Maria Miklowski, MD, FACP

Department of Hospital Medicine, Cleveland Clinic Community Care, Cleveland Clinic; Clinical Assistant Professor, Cleveland Clinic Lerner College of Medicine of Case Western Reserve University, Cleveland, $\mathrm{OH}$
Bruce Jansen, MD

Department of Hospital Medicine, Cleveland Clinic

Community Care, Cleveland Clinic; Clinical Assistant

Professor, Cleveland Clinic Lerner College of Medicine of Case Western Reserve University, Cleveland, $\mathrm{OH}$
Moises Auron, MD, FAAP, FACP, SFHM Department of Hospital Medicine, Cleveland Clinic Community Care Cleveland Clinic; Associate Professor, Cleveland Clinic Lerner College of Medicine of Case Western Reserve University, Cleveland, $\mathrm{OH}$

Christopher Whinney, MD, FACP, SFHM

Chairman, Department of Hospital Medicine, Cleveland Clinic Community Care, Cleveland Clinic; Clinical Assistant Professor, Cleveland Clinic Lerner College of Medicine of Case Western Reserve University, Cleveland, $\mathrm{OH}$

\title{
The hospitalized patient with COVID-19 on the medical ward: Cleveland Clinic approach to management
}

\section{Posted October 27, 2020}

\section{ABSTRACT}

SARS-CoV-2-infected inpatients who are admitted to a noncritical care medical ward require a standardized approach that is based on evidence if available, and effective supportive and respiratory care. Outcomes are better when patients receive standardized care, in special COVID-19 wards in the hospital, from clinical teams with expertise. Available evidence and guidelines should be continuously appraised and integrated into clinical protocols for all domains of treatment, including isolation, and personal protective measures, pharmacologic therapy, and transitions of care. Inpatient pharmacologic therapy at this time consists primarily of dexamethasone and remdesivir, along with thromboprophylaxis, given the coagulopathy associated with COVID-19. This article summarizes current practices in our organization.

\section{STANDARDIZED APPROACH NEEDED}

Throughout the COVID-19 pandemic, there has been an onslaught of data, often conflicting and difficult to interpret for the clinician on the frontlines. A dearth of solid evidence has led at times to ad hoc practices or extrapolation from poor-quality studies, as the medical community worldwide meets the challenge of combating a novel infectious disease.

Disclosures: The authors report no relevant financial relationships which, in the context of their contributions, could be perceived as a potential conflict of interest.

Disclaimer: The statements and opinions expressed in COVID-19 Curbside Consults are based on experience and the available literature as of the date posted. While we try to regularly update this content, any offered recommendations cannot be substituted for the clinical judgment of clinicians caring for individual patients.

doi:10.3949/ccjm.87a.ccc064
However, strict adherence to standard supportive and respiratory care remains the mainstay of treatment, as in other viral and respiratory diseases. Standardized admission orders and templates for progress notes, along with a team-based approach, provide support to the clinician and enhance the quality of care and safety of our patients.

This is a review of high-yield practical care recommendations for patients with COVID-19 admitted to general medicine wards.

\section{CLINICAL PRESENTATION}

The common signs and symptoms of COVID-19 are similar to those of other viral infections: fever, chills, shortness of breath, cough, myalgia, headache, fatigue, sore throat, rhinorrhea, diarrhea, nausea, and vomiting. Anosmia and ageusia have also been reported as prominent clinical features. Hospitalized patients more commonly present with dyspnea, fever, and cough. ${ }^{1,2}$ Elderly adults may present with atypical features such as confusion alone. An unusual clinical tolerance to hypoxia may be seen, patients often denying shortness of breath and showing no sinus tachycardia despite needing significant oxygen supplementation.

The incubation period or median time to symptom onset from exposure is 4 to 5 days, though it can be up to 14 days. $^{3}$ In an early study in 1,099 patients in China, ${ }^{4} 88.7 \%$ of patients developed fever while in the hospital, $67.8 \%$ had cough, and 3.8\% had diarrhea. The median length of stay was 12 days (mean 12.8), 3.4\% of patients had acute respiratory distress syndrome (ARDS), and 1.1\% developed shock. In 44,672 patients in China, ${ }^{5}$ most cases (81\%) were mild (with no pneumonia, or mildly symptomatic 
with pneumonia), 14\% were severe (with dyspnea, increased respiratory rate, arterial oxygen saturation $\left[\mathrm{SaO}_{2}\right] \leq 93 \%$, or ratio of partial pressure of arterial oxygen to fraction of inspired oxygen $\left[\mathrm{PaO}_{2} / \mathrm{FiO}_{2}\right]<$ 300 ), and $5 \%$ were critical (with respiratory failure, shock, or multiorgan system dysfunction or failure).

\section{Clinical progression during hospitalization}

The median time from symptom onset to developing dyspnea is 5 to 8 days; to developing ARDS 8 to 12 days, and to intensive care unit (ICU) admission is 10 to 12 days. Among hospitalized patients with severe disease, $26 \%$ to $32 \%$ were admitted to the ICU, and $67 \%$ to $85 \%$ of the COVID-19 ICU patients developed ARDS, with ICU mortality rates ranging from $39 \%$ to $72 \%$. The median length of stay in the hospital was 10 to 13 days for survivors. ${ }^{6}$

\section{CLINICAL RISK FACTORS FOR DISEASE AND SEVERITY OF ILLNESS}

Among 191 patients at Wuhan Pulmonary Hospital treated before January 31, 2020,7 risk factors present at the time of admission that predicted death included:

- Older age (odds ratio [OR] 1.10 per year increase, 95\% confidence interval [CI] 1.03-1.17, P $=.0043$ )

- Higher Sequential Organ Failure (SOFA) score (median score 1.0 in survivors vs 4.5 in nonsurvivors, OR 5.65, 95\% CI 2.61-12.23, P <.0001)

- D-dimer level greater than $1,000 \mathrm{ng} / \mathrm{mL}(1 \mu \mathrm{g} /$ mL) (OR 18.42, 95\% CI 2.64-128.55, $\mathrm{P}=.0033$ ).

Elevated blood levels of interleukin 6, high-sensitivity cardiac troponin I, and lactate dehydrogenase and lymphopenia were associated with more severe COVID-19 illness.?

In view of concern for more severe illness in pregnancy, ${ }^{8}$ the US Centers for Disease Control and Prevention surveyed 8,207 pregnant patients with COVID-19 from January through June 2020. Pregnant patients were more likely to need ICU admission (adjusted relative risk [aRR] 1.5, 95\% CI 1.2-1.8) and to need mechanical ventilation (aRR 1.7, 95\% CI 1.2-2.4), while the mortality rate was essentially the same at $0.2 \% .{ }^{9}$ The Pregnancy Coronavirus Outcomes Registry (PRIORITY) study investigate the effects of COVID-19 on pregnant and postpartum women and will help inform recommendations for patients and health care workers.

Data are still conflicting on risk in patients with autoimmune diseases or receiving immunosuppressive treatment. ${ }^{10}$

\section{ADMISSION CRITERIA}

Major guidelines have set no firm criteria for admission to the hospital, and the decision can be affected by local disease prevalence, community resources, and hospital capacity. Thresholds for admission may be lower, for instance, when a hospital is not at capacity and when community resources are robust.

At our institution, we admit patients who have evidence of moderate to severe disease, manifested by dyspnea at rest, hypoxia (oxygen saturation on room air $<94 \%$ ), high fevers, diarrhea, altered mental status, failure to thrive, or inability to manage symptoms at home.

Escalation to ICU-level care is typically due to worsening dyspnea, deterioration in respiratory status resulting in escalating oxygen requirements (eg, high-flow oxygen, noninvasive positive pressure ventilation, or intubation), shock, or strong inflammatory response (cytokine storm).

\section{DIAGNOSIS}

At Cleveland Clinic, all patients admitted to the hospital or who plan to undergo procedures or surgery are tested for COVID-19. We use either a rapid test for viral antigen or a standard reverse transcription polymerase chain reaction (RT-PCR) test to detect SARS-CoV-2 RNA in nasopharyngeal samples. The rapid antigen tests are less sensitive than RT-PCR tests, and range from 84\%-97.6\% with reported specificity of $100 \%$. Retesting may be indicated if the pretest probability and clinical suspicion for COVID-19 is high but the test result is negative. ${ }^{11}$ The sensitivity of RT-PCR tests may be affected by the duration of illness. Peak time of testing to minimize false negatives in one validation study on RTPCR for SARS-CoV-2 was 3 days following symptom onset (correlating to day 8 of infection). The quality of the sample obtained (depth of nasopharyngeal swab and time swab left in place) also impacts test sensitivity. ${ }^{12-13}$

The patient's presenting symptoms and abnormalities on laboratory tests and imaging commonly obtained during admission might increase suspicion of SARS-CoV-2 infection before the screening test results are received. Lymphopenia, leukocytosis, elevations in aspartate aminotransferase, alanine aminotransferase, C-reactive protein, erythrocyte sedimentation rate, and D-dimer and a low to normal procalcitonin level may all suggest COVID-19.

Chest imaging (radiography and computed tomography) may show ground-glass opacities or 
other findings suggesting a viral respiratory infection. ${ }^{14}$ Elements of the patient's clinical presentation, in combination with these findings, may suggest the patient should be considered a "patient under investigation" on admission and triaged to the appropriate isolation until the screening results return.

\section{TREATMENT}

Active treatment of patients with suspected or confirmed COVID-19 must be supported by evidence from randomized controlled trials whenever possible. However, when evidence is lacking, some decisions must be made on the basis of clinical judgment, small or imperfect studies, observational data, and expert opinion. We believe the most relevant and consistent approach is to strictly adhere to a bundle of care that encompasses proper isolation criteria, formal and careful use of personal protective equipment, and respiratory supportive care.

We have several recommendations for practical care of hospitalized patients with COVID-19.

\section{Dedicated COVID-19 units}

We endorse admitting hospitalized patients with COVID-19 to special units, for several reasons:

- To isolate and contain active cases

- To mitigate the risk of transmission within the hospital

- To allow effective standardization and practicality of care.

On these units, a team of healthcare providers (nurses, nurse's aides, respiratory therapists, physicians, advanced practice providers, and environmental services and dietary staff) undergoes continuous training and develops expertise in the care of these patients, enhancing quality and patient safety.

\section{Team organization and development}

The time required for daily patient care is affected by several factors:

- Donning and doffing of personal protective equipment, initially using a buddy system to assure it is done properly

- Rapid changes in patient acuity and clinical deterioration requiring undivided clinical attention

- Continuous medical education due to the rapidly evolving developments in the field.

At our institution's large academic medical center, physicians are paired with advanced practice providers. This allows for division of labor, including documentation, assuring use of standardized admission order sets and discharge navigation tools that trigger patient tracking after discharge. At our community hospitals, where the COVID-19 admission census may be smaller, a sole physician may provide medical care. The advanced practice provider can also serve as a liaison to families, who are restricted from visiting except in special circumstances (eg, end-of-life care, day of surgery), as well as with the infectious disease consultants who are the most commonly called-upon colleagues in caring for these patients.

Our main campus and community hospitals ensure harmonization of care by having regular hospital medicine and primary care educational meetings with both our department chair and site directors, and regular noon conference continuing medical education updates. There is a central repository for care protocols on our intranet that is shared with hospitalists by email at the start of a rotation on a COVID-19 ward service. We hold a "plan-of-care" visit in which the nurse, provider team, and patient communicate directly. Clinicians are trained on the protocols of a physician-advanced practice provider pair team before they work on the COVID-19 unit. Regular communication with the patient's family occurs by phone, and we have a tablet device for patients to use to connect with their families using standard online communication tools.

We recommend creating teams of dedicated providers with a sufficient breadth of expertise, such as hospitalists, who can rotate off service to other assignments to allow for some recovery from the stresses of a busy COVID-19 service. Leadership teams should solicit feedback from the frontline caregivers and check in on their wellbeing either directly or through surveys.

Very few employees working in the COVID-19 units at Cleveland Clinic have become infected; their infection rates have paralleled those in the community, suggesting that they acquired SARSCoV-2 through community spread rather than in the hospital. At this time, there are no guidelines regarding routine testing for hospitalists or nurses in the absence of symptoms after working on the inpatient COVID-19 service. Guidance on masking or social distancing from the caretaker's families after spending time on a COVID-19 ward is also lacking, and neither intervention is routinely recommended. As some studies have found that COVID-19 is more severe in pregnant women, pregnant staff, nurses, and other support staff should not be assigned on these units until more definitive work is done to clarify risk.

Cleveland Clinic's Office of Professional Staff Affairs offers a range of caregiver support, such as 
TABLE 1

Cleveland Clinic COVID-19 non-ICU order set

\begin{tabular}{|c|c|}
\hline Type & Orders \\
\hline Respiratory & $\begin{array}{l}\text { Oxygen management } \\
\text { Oxygen device: [eg, nasal cannula] } \\
\text { Flow } \\
\text { Target } \mathrm{SpO}_{2} \text { range }\end{array}$ \\
\hline Laboratory tests & 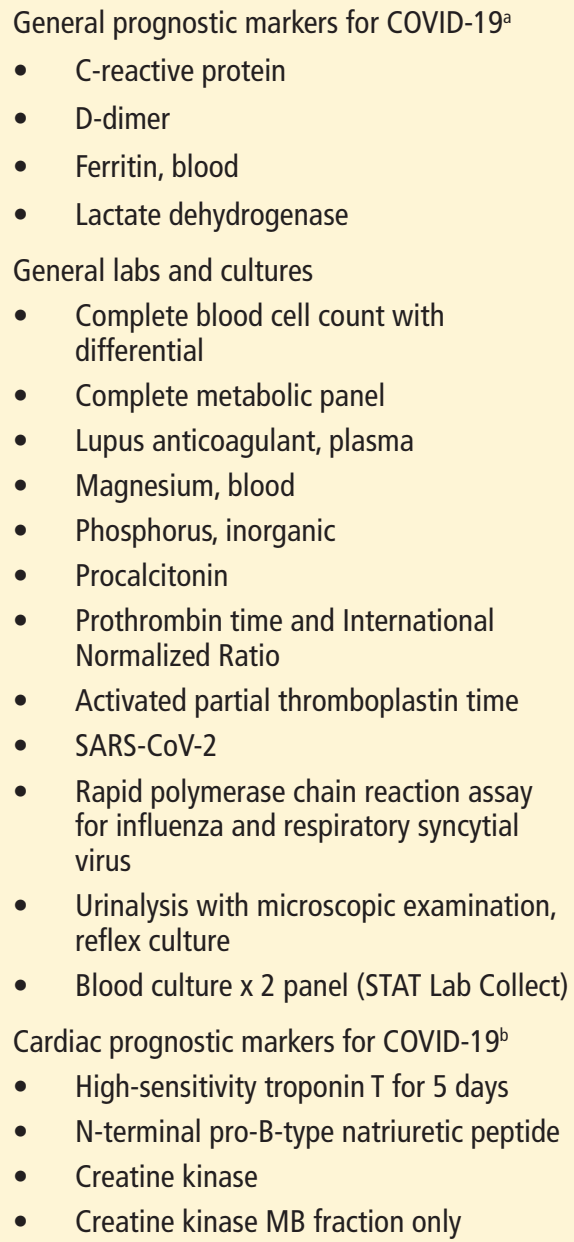 \\
\hline Imaging ${ }^{c}$ & $\begin{array}{l}\text { Chest radiograph } 2 \text { views (front and lateral) } \\
\text { Chest radiograph } 1 \text { view (front, portable) }\end{array}$ \\
\hline Cardiology & Electrocardiography \\
\hline
\end{tabular}

a Inflammatory markers (ferritin, lactate dehydrogenase, D-dimer, C-reactive protein) may be tracked daily or every other day initially.

${ }^{b}$ Severely elevated cardiac markers, without acute ischemic changes on an electrocardiogram, suggest inflammatory injury rather than acute coronary syndrome.

cPortable imaging is preferred to reduce caregiver exposure. web-based guidance and support phone calls, as well as family care resources. Support services for SARS-CoV-2-positive caregivers may include meal delivery, connecting to child and elder care services, wellbeing apps, virtual programs and chats, as well as prescription delivery and access to a Care Support Team liaison.

\section{Personal protective equipment}

Patients should be asked to wear a surgical or ear-loop mask whenever a caregiver is in the room. Universal masking in the hospital has been shown to significantly decrease the transmission of SARS-CoV-2. ${ }^{15}$

Providers may elect to obtain a history from the patient by phone or video to limit their exposure, although we have not routinely done this in our practice during this phase of the pandemic. If feasible, consultants may also conduct visits by phone or video to preserve personal protective equipment and limit risk to additional staff. We have also piloted a "tele-admitter" role to cope with a potential surge in cases and to limit inroom time for clinicians. This provider can take a patient's history by phone, and a second provider visits the patient for a brief physical examination and consolidation of the care plan.

A surgical or ear-loop face mask, a face shield, gloves, and a gown are recommended for routine visits with patients with COVID-19 at our institution. Cleveland Clinic recommends using an N95 mask during aerosol-generating procedures (during which a large number of virus particles can be released and linger in the air) such as bronchoscopy, endoscopic procedures, transesophageal echocardiography, noninvasive positive-pressure ventilation, use of high-flow oxygen, open tracheal suctioning, nasoenteric tube placement, intubation, extubation, and cardiopulmonary arrest and resuscitation. Although SARS-CoV-2 can be aerosolized and persist on surfaces, most infections appear to be transmitted through the droplet route. ${ }^{16,17}$ To date, no robust trials have shown N95 masks to be superior to surgical masks when caring for patients with viral infections that are primarily transmitted by droplets. ${ }^{18}$ On COVID19 wards, a clinician may use the same face mask and eye protection during repeated close contacts with different patients, without removing them between patients. ${ }^{19,20}$ We have some gowns that can be washed and face shields and N95 masks that can be decontaminated and reused. 


\section{Code status discussion}

It is important to discuss code status and goals of care with patients with COVID-19 early on. A patient's respiratory status can deteriorate with stunning swiftness, leaving little time for a meaningful discussion during the ensuing crisis.

\section{Laboratory monitoring}

Inflammatory and cardiac markers have been shown to aid in assessing disease severity, following response to treatment, defining prognosis, and guiding treatment in a variety of diseases, including COVID-19. ${ }^{21}$ We have developed a set of orders in the electronic medical record to guide clinicians in monitoring trends in a patient's biochemical status and recognizing the development of progressive inflammation and end-organ damage. Table 1 shows a sample of the main features of our order set. These are prompts that can be selected individually by the provider.

\section{Medications}

Bronchodilators. We do not order nebulized medications, in view of the risk of viral aerosolization. If bronchodilators are needed, we prescribe them in inhalers, eg, albuterol inhaler with a spacer, 4 puffs per dose, rather than nebulized albuterol. It is practical to keep this medication at the bedside of any patient who may need it, to avoid delay in giving it and to avoid requiring a respiratory therapist to return to the room to administer it.

Nonsteroidal anti-inflammatory drugs (NSAIDs). Although the Health Minister of France warned against the use of NSAIDs due to an increased risk of severe adverse events, ${ }^{22}$ a World Health Organization review subsequently showed no adverse effects in this setting, and chronic NSAIDs may be continued in patients already taking them if there are no contraindications. ${ }^{23}$ However, acetaminophen is still preferred to NSAIDs for fever control, in view of the known adverse effects of NSAIDs such as gastrointestinal complications, bleeding, and renal compromise.

Angiotensin-converting enzyme (ACE) inhibitors and angiotensin II receptor blockers (ARBs). SARS-CoV-2 enters epithelial lung cells via angiotensin-converting enzyme 2 (ACE2) receptors, and early in the pandemic some suggested that ACE inhibitors and ARBs, which up-regulate ACE2 expression, might be either beneficial or harmful in COVID19. Since that time, multiple medical associations, including the American College of Cardiology, the Heart Failure Society of America and the American Heart Association have jointly issued a strong recom- mendation against discontinuing these medications. ${ }^{24}$ Our practice is to continue them unless other medical contraindications exist.

Statins. Although COVID-19 may cause new elevations in liver enzymes, no studies to date have demonstrated adverse effects from continuing prescribed statins in these patients. They are often an important component of treatment for underlying cardiovascular diseases and should be continued. A meta-analysis of 4 studies with a total of 8,990 patients with COVID-19 suggested a 30\% reduction in fatal or severe disease with statins. ${ }^{25}$ Randomized controlled trials are necessary to confirm these findings and to suggest a dosing protocol. We continue statins unless other contraindications exist.

Glycemic control. In 7,337 patients with COVID19 in Hubei Province in China, those who had diabetes at admission had a higher mortality rate, while well-controlled inpatient glucose levels correlated with lower levels of inflammatory markers and better clinical outcomes. The mortality rate in patients with diabetes was $7.8 \%$, compared with $2.7 \%$ in those without diabetes, adjusted hazard ratio [aHR] 1.49 . Well-controlled inpatient glucose, with low glycemic variability, was associated with significantly lower mortality, aHR $0.14 .{ }^{26}$

While insulin remains the preferred and recommended medication for most hospitalized diabetic patients, basal-bolus regimens for patients who are in isolation will require the nurses to spend more time in the patient's room and to use more personal protective equipment. Consider allowing select patients to check their own blood sugars with their home glucometer or continuous glucose monitoring system and report them to the nurse. We recognize this confers some risk, including generating many data points to act upon if a patient has a continuous glucose monitoring system.

Clinicians should consider continuing oral medications that carry a lower risk of hypoglycemia, such as metformin, unless otherwise contraindicated. Initial advisories against the use of metformin based on a theoretical risk of lactic acidosis or hepatic dysfunction have later been challenged by studies showing a beneficial effect in continuing the drug. Potential pleotropic antiviral and anti-inflammatory effects may be responsible for this salutary effect, but definitive answers from randomized controlled trials are lacking. ${ }^{27}$ Others have recommended sitagliptin as an oral agent in this setting, which poses a lower risk of hypoglycemia and avoids the sole use of insulin sliding scale regimens. ${ }^{28}$ 
Select patients may also be able to use their own insulin pens. This will be new to the practice of inpatient medicine for many but may be more practical and patient-centered.

If insulin infusions are needed, the intravenous tubing can be extended to a pump placed outside the door, a practice already employed in many COVID19 cohort ICUs to avoid frequent trips in and out of the patient's room.

Antibiotics. COVID-19 can occur in the setting of other bacterial infections or become complicated by bacterial superinfection. Diagnostic reasoning aided by persistent or worsening inflammatory markers such as procalcitonin, leukocytosis, an abnormal urinalysis, or worsening findings on chest imaging may suggest a bacterial infection that requires antibiotic therapy. If uncertain, it may be prudent to start antibiotic therapy, de-escalating quickly if blood, sputum, and urine cultures are negative. A normal to low procalcitonin level may also aid in the decision to discontinue antibiotics.

\section{Supportive care}

Incentive spirometry. Though there are no randomized controlled trials in nonventilated patients with COVID-19, we routinely give them an incentive spirometer to use every hour while awake, and "often" after discharge. It may be beneficial, it is inexpensive and easy, and it allows patients to participate in their care and recovery.

Pulse oximetry. Consider continuous monitoring at admission if a patient complains of dyspnea and if they need supplemental oxygen, or in patients with dementia or delirium who may not be able to selfreport symptoms of dyspnea.

Telemetry. There is no indication for continuous telemetry unless cardiac injury is manifest by symptoms, electrocardiographic changes, arrhythmias, or troponin elevations. Telemetry may be an expensive, unnecessary allocation of resources and often triggers alarms in patients at low risk, increasing the burden on nurses who must return numerous times to adjust leads and monitor equipment.

Prone positioning. Although used more routinely in the treatment of moderate-to-severe ARDS, prone positioning has been considered for non-intubated floor patients with COVID-19. It may improve oxygenation and gas exchange compromised by alveolar collapse with prolonged supine positioning. It may also improve management of secretions. ${ }^{29}$

We do not have a floor protocol for this at our institution, but it is reasonable to place patients in the prone position 1 hour at a time, as much as tolerated, moving to lateral decubitus positions periodically, or if not tolerated, have them sit up in bed rather than lying flat, and ambulate in their room if able.

In a study of 29 patients with confirmed COVID19 admitted to a New York City step-down unit with severe hypoxemic respiratory failure, ${ }^{30} 19$ patients had an $\mathrm{SaO}_{2}$ of $95 \%$ or greater at the end of an hour in the prone position. Seven patients ultimately required intubation. Of the 6 patients who had an $\mathrm{SaO}_{2}$ less than $95 \%$ after an hour, 5 (83\%) were intubated.

In cases of rapid deterioration, this treatment should not be used in an attempt avoid intubation, as delay in intubation would be deleterious. Patients placed in the prone position should not have altered mentation and must be able to mobilize without assistance.

Expectorants, mucolytics. We recommend therapies such as guaifenesin as needed to manage secretions. We also avoid aerosolized therapies.

Intravenous fluids. We advise against aggressive fluid administration and giving maintenance fluids. The reason is to avoid possible volume overload due to the risk of ARDS in these patients, in whom a more volume-restrictive approach is generally advised.

\section{Radiographic studies}

We avoid sending patients off the floor unnecessarily for imaging studies, unless clinically imperative, such as to evaluate emergently for venous thromboembolism.

\section{Venous thromboembolism prophylaxis}

COVID-19 results in a hypercoagulable state. The incidence of deep vein thrombosis and pulmonary embolism as well as arterial thrombosis in patients with COVID-19 is elevated as much as 30\%, especially in those with severe disease. Venous thromboembolism prophylaxis should be ordered for all patients with COVID-19. ${ }^{31-33}$ The current thromboprophylaxis protocol at Cleveland Clinic involves subcutaneous enoxaparin or heparin based on renal function, at standard prophylactic doses for less-ill patients and at twice-daily or intermediate prophylactic dosing for those with D-dimer levels higher than 3,000 ng/mL or critical illness (Table 2).

In a retrospective study of 4,389 patients with COVID-19 admitted to New York City hospitals, the use of anticoagulation, in prophylactic or therapeutic doses, was associated with lower rates of mortality and intubation. ${ }^{34}$ Prospective trials are needed to confirm these findings and establish the most effective dosing regimens. 
TABLE 2

\section{Thromboprophylaxis for patients with COVID-19 or patients under investigation}

If D-dimer is $<3,000 \mathrm{FEU}$ or unknown, and:

- No renal impairment: enoxaparin $40 \mathrm{mg}$ every 24 hours

- Creatinine clearance 10-30 mL/hour: enoxaparin $30 \mathrm{mg}$ every 24 hours

- Creatinine clearance $<10 \mathrm{~mL} /$ hour, acute kidney injury, or dialysis: heparin 5,000 units every 12 hours

If D-dimer is $\geq 3,000 \mathrm{FEU}$ or critically ill patient

(independent of D-dimer level), and:

- No renal impairment: enoxaparin $40 \mathrm{mg}$ every 12 hours

- Creatinine clearance 10-30 mL/hour: enoxaparin $40 \mathrm{mg}$ every 24 hours

- Creatinine clearance $<10 \mathrm{~mL} / \mathrm{hour}$, acute kidney injury, or dialysis: heparin 7,500 units every 12 hours

We have also used extended prophylaxis after discharge in select patients with high D-dimer levels and low bleeding risk based on clinical risk stratification tools. ${ }^{35}$ This important issue is more fully discussed in a recent review article in this journal. ${ }^{36}$ American College of Chest Physician guidelines recommend low-molecular-weight heparin over unfractionated heparin for thromboprophylaxis at standard doses (not intermediate twice-a-day or weight-based escalated doses). ${ }^{37}$ In the same vein, these guidelines address the clinical question of prescribing extended thromboprophylaxis in patients with COVID-19, recommending it in patients with low bleeding risk "if emerging data on the postdischarge risk of venous thromboembolism and bleeding indicate a net benefit of such prophylaxis." ${ }^{37}$

\section{Therapies}

Dexamethasone, a glucocorticoid, is given at a dose of $6 \mathrm{mg}$ intravenously or by mouth daily for up to 10 days.

The Randomized Evaluation of Covid-19 Therapy (RECOVERY) trial was a randomized controlled trial in the United Kingdom comparing dexamethasone $6 \mathrm{mg}$ intravenously or by mouth vs usual care in patients with COVID-19 hospitalized either on no supplemental oxygen, oxygen, or mechanical ventilation; the mortality rate at 28 days was the primary outcome. The mortality rate was lower in those receiving dexamethasone, with the difference most pronounced in patients on mechanical ventilation
( $29.3 \%$ vs $41.4 \%$, rate ratio $0.64,95 \%$ CI $0.51-0.81$, followed by those requiring supplemental oxygen (23.3\% vs $26.2 \%$, rate ratio $0.82,95 \%$ CI $0.72-0.94)$. The mortality rate was numerically higher with dexamethasone than without it in the subgroup not receiving supplemental oxygen, but the difference was not statistically significant $(17.8 \%$ vs $14 \%$, rate ratio $1.19,95 \% \mathrm{CI} 0.91-1.55) .^{38}$

In practice, dexamethasone is prescribed more frequently than remdesivir in patients with COVID. 19 , as it is readily available and does not require prior approval from an infectious disease specialist. We prescribe dexamethasone for all COVID-19 inpatients who need oxygen supplementation (oxygen saturation $<94 \%$ on room air).

A prospective meta-analysis sponsored by the World Health Organization found that dexamethasone, hydrocortisone, and methylprednisolone all decreased 28-day all-cause mortality in critically ill patients with COVID-19, suggesting these benefits are likely a class effect and do not depend on the use of a specific glucocorticoid. The comparison of lowdose vs high-dose corticosteroids among the 7 studies in the meta-analysis was noted to be imprecisely estimated, and there was no suggestion of increased risk of serious adverse events in the corticosteroid groups. ${ }^{39}$

Remdesivir is an intravenous antiviral nucleotide analogue with activity against SARS-CoV-2. It is likely most effective when prescribed to patients with mild to moderate pulmonary disease within the first 10 days of symptoms, as this is the time of greatest viral replication. It is given for 5 days, with a $200-\mathrm{mg}$ intravenous loading dose on day 1 , followed by 100 mg intravenously daily for 4 days. For critically ill or intubated patients, the $100 \mathrm{mg}$ intravenous daily dose may be extended to 9 days.

The Adaptive Covid-19 Treatment Trial (ACTT1),,$^{40}$ a randomized, double-blind, multinational study, showed a faster median time to recovery, the modified primary end point (11 days in the placebo group vs 15 days in the remdesivir group), with a rate ratio for recovery of 1.29 (95\% CI 1.12-1.49, P < .001). Remdesivir also improved clinical condition at day 15 (the study's original primary end point before the disease course was found to be more protracted than expected), defined as discharge from hospital or improvement on an 8-point ordinal scale identifying need for oxygen and other supportive medical care (odds ratio for benefit 1.5, 95\% CI 1.2-1.9, P = .001 ). The benefit was more pronounced for patients with a higher ordinal scale and need for supplemental 
oxygen. The mortality rate was lower with remdesivir but the difference was not statistically significant. Serious adverse events were noted in $24.6 \%$ of those in the remdesivir group compared with $31.6 \%$ in the placebo group.

Contraindications include an estimated glomerular filtration rate less than $30 \mathrm{~mL} / \mathrm{min} / 1.73 \mathrm{~m}^{2}$ or alanine aminotransferase level greater than 5 times upper limit of normal. Liver enzyme levels should be followed daily during a course of treatment.

A randomized, open-label trial of remdesivir in hospitalized patients with mild to moderate disease (pulmonary infiltrates and a pulse oxygen saturation $>94 \%$ on room air) showed a significant improvement in clinical status compared with routine care at 11 days after initiation of treatment. A 5-day course showed a benefit in this population, while a 10-day course did not. ${ }^{41}$ These findings are of uncertain clinical significance, as most of these patients would probably not meet the criteria for hospital admission in most settings.

However, on August 28, 2020 the US Food and Drug Administration authorized the use of remdesivir for all hospitalized patients with suspected or confirmed COVID-19, regardless of disease severity. ${ }^{42}$ Remdesivir is still most commonly used in those with severe disease with an oxygen requirement $\left(\mathrm{SpO}_{2}<\right.$ $94 \%$ on room air). At our institution, this medication must be ordered by an infectious disease consultant. After consideration of the expected clinical benefits of a course of remdesivir, decisions must still be made on a case-to-case basis rather than using a blanket protocol covering all hospitalized patients with COVID-19.

On October 22, 2020, the FDA approved remdesivir for use in all patients 12 years of age and older and weighing at least $40 \mathrm{~kg}$ requiring hospitalization for COVID-19.43

Convalescent plasma is obtained from patients who have recovered from COVID-19 and have detectable levels of antibodies.

Early data from very small case series, involving 5 and 10 patients, suggested a possible benefit from convalescent plasma. ${ }^{44,45}$ In a randomized controlled trial published in June 2020, in 103 participants, there was no significant difference in time to clinical improvement between those treated with convalescent plasma vs usual care. Further analysis showed that there may have been some benefit for those with severe disease, but the sample size was small. ${ }^{46}$

Another study showed that plasma doses with higher antibody levels were more effective than doses with lower antibody levels and earlier treatment (within 3 days of diagnosis) was more effective than later treatment ( 4 or more days after diagnosis). The authors concluded that this data could help inform the design of future prospective trials of convalescent plasma use in patients with COVID-19.47 The study was not peer-reviewed and had no placebo group, but on August 23, 2020, the FDA granted an emergency use authorization for convalescent plasma in all hospitalized patients with COVID-19.

While we wait for convincing data from randomized controlled trials regarding the potential benefits of convalescent plasma in patients with COVID-19, decisions on its use are made on a case-to-case basis and, at Cleveland Clinic facilities, require an infectious disease consult.

Research and investigational therapies. Cleveland Clinic has an active inpatient research team that has been reviewing our admitted patient lists to identify patients with COVID-19 since the onset of the pandemic. The team has a daily huddle to assess patient eligibility for clinical trials. They hold regular virtual meetings with the Department of Hospital Medicine with updates on the ongoing trials, and work with the hospitalist or intensivist caring for the patient.

There are numerous ongoing trials worldwide including evaluations of interferon plus remdesivir, baricitinib (a Janus kinase inhibitor), sarilumab (an IL-6 inhibitor), infliximab (a tumor necrosis factor inhibitor), and others. ${ }^{48}$

\section{DISCHARGE}

We use several criteria when discharging a COVID19 patient. The patient should be afebrile for 24 to 48 hours, off supplemental oxygen or have a stable oxygen requirement for 2 to 3 days, and have evidence of declining inflammatory markers. Ideally, they should be past the period when respiratory deterioration is most likely to occur ( 8 to 12 days since symptom onset), but this is not strictly necessary if the patient is otherwise doing well and can be closely monitored as an outpatient, including regular pulse oximetry checks in those at high risk due to comorbidities. The patient may continue using an incentive spirometer while at home. Detailed discharge instructions for patients wtih COVID-19 are imperative to transition care back to their community primary care physicians and other members in their health team.

We work closely with the case management and social work departments to identify local skilled nursing facilities and long-term care facilities that have the ability to care for patients convalescing with COVID- 
19 after discharge. Criteria vary for these facilities, and some require repeat testing to triage patients to a COVID or non-COVID wing. These facilities are able to accept patients on supplemental oxygen. One of our own hospital-based skilled nursing facilities has converted a floor for patients with COVID-19 able to be discharged, helping keep inpatient beds available for those most in need of hospital-level care.

Cleveland Clinic has a robust home-monitoring program that reaches out to patients after discharge. The details of this program are outlined elsewhere in this journal. ${ }^{49}$ This supports our patients after discharge and helps decompress the burden of readmissions. In our region, the Ohio Department of Health also follows patients with COVID-19 and makes the ultimate determination when the patient can end isolation. The current Centers for Disease Control and Prevention guideline recommends deisolation using a symptom-based approach: 10 days from symptom onset, 24 hours since last fever without use of a fever-reducing medication, and symptom improvement. Isolation for up to 20 days for patients who have recovered from severe illness may be warranted. ${ }^{50}$ Extended venous thromboembolism prophylaxis for up to 5 weeks may be recommended for some patients, including those with an IMPROVE score of 5, IMPROVE score of 3 and D-dimer level higher than 1,000 at discharge, or positive antiphospholipid antibodies and D-dimer higher than 1,000 at discharge. ${ }^{51,52}$ More studies are needed to fully refine this recommendation. ${ }^{37}$

\section{CONCLUSION}

COVID-19 may be the next "great imitator," protean in manifestations and complications. On the one hand, given its continued rise in prevalence and highly variable presentation, SARS-CoV-2 infection should almost always be in the differential diagnosis when evaluating a patient at this time. However, our patients will still present with the same conditions they had in the past, some requiring immediate treatment that we might be tempted to forego if we assume the patient is suffering from this viral illness alone.

Other than excellent supportive care, our treatment options remain limited, but at least two medications have been shown to be helpful, dexamethasone and remdesivir (Table 3). Keeping abreast of the public health data and guidelines that are the watchdogs of emerging and useful therapies will be as relevant to this disease process as it is in other areas of practice. ${ }^{53-55}$
TABLE 3

\section{Summary of treatment recommendations for patients with COVID-19 at Cleveland Clinic}

Mild to moderate illness (no hypoxia, no radiographic pneumonia, oxygen saturation $\left(\mathrm{SpO}_{2}\right)>94 \%$ on room air)

Supportive care

Severe illness (hypoxia $\left[\mathrm{SpO}_{2}<94 \%\right.$ on room air], radiographic evidence of pneumonia, not critically ill)

Supportive care

Dexamethasone

Remdesivir

Infectious disease consult

Possibly emerging therapies

Possible enrollment in clinical trials

Critically ill (mechanically ventilated)

Dexamethasone

Remdesivir

Infectious disease consult

Possibly convalescent plasma (in the context of a clinical trial)

Possibly emerging therapies

Possible enrollment in clinical trials

Immunosuppressed patients

Consider reduction in immunosuppression in conjunction with rheumatology or oncology consultant

Dexamethasone

Remdesivir

\section{Pregnant patients}

As above, consider risks and benefits of glucocorticoids and remdesivir

Medicine has always been a discipline requiring continuous evaluation of engrained practices and novel treatments. We have been willing to change when former strongly held beliefs turned out to be false (such as touting estrogen as cardioprotective or recommending full bedrest for those with deep vein thrombosis). In the past, these changes came slowly, even reluctantly. But now, during the COVID-19 pandemic, changes in recommendations for everything from masking precautions to treatment regimens occur at lightning speed. Complicating the matter, this process has been public and revealed a not-so-closely guarded secret for all to see-we do not know everything. Vaccines will arrive. Changes in knowledge and treatments will continue. If we stay agile, keep up with evidence-based recommendations, 
and maintain strict adherence to supportive therapy guidelines, we will continue to offer our patients the highest quality care.

\section{REFERENCES}

1. CDC COVID-19 Response Team. Severe outcomes among patients with coronavirus disease 2019 (COVID-19) - United States, February 12-March 16, 2020. MMWR Morb Mortal Wkly Rep 2020; 69(12):343-346. doi:10.15585/mmwr.mm6912e2

2. CDC COVID-NET. A weekly summary of U.S. COVID-19 hospitalization data. Preliminary data as of Aug 8, 2020. Accessed 8/21/2020. https://gis.cdc.gov/grasp/COVIDNet/COVID19_5.html

3. Lauer SA, Grantz KH, Bi Q, et al. The incubation period of coronavirus disease 2019 (COVID-19) from publicly reported confirmed cases: estimation and application. Ann Intern Med 2020; 172(9):577-582. doi:10.7326/M20-0504

4. Guan WJ, Ni ZY, Hu Y, Liang WH, Ou CQ, He JX, et al. Clinical characteristics of coronavirus disease 2019 in China. N Engl J Med (2020) 382:1708-20. doi: 10.1056/NEJMoa2002032

5. Wu Z, McGoogan JM. Characteristics of and important lessons from the coronavirus disease 2019 (COVID-19) outbreak in China: summary of a report of 72,314 cases from the Chinese Center for Disease Control and Prevention. JAMA 2020; 323(13):1239-1242. doi:10.1001/jama.2020.2648

6. Hospitalization rates and characteristics of patients hospitalized with laboratory confirmed coronavirus disease 2019. https://www. cdc.gov/coronavirus/2019-ncov/hcp/clinical-guidance-managementpatients.html

7. Zhou F, Yu T, Du R, et al. Clinical course and risk factors for mortality of adult inpatients with COVID-19 in Wuhan, China: a retrospective cohort study [published correction appears in Lancet 2020; 395(10229):1038]. Lancet 2020; 395(10229):1054-1062. doi:10.1016/ S0140-6736(20)30566-3

8. ACOG Statement on COVID-19 and Pregnancy. Jun 24, 2020. Accessed on 8/21/2020. https://www.acog.org/news/news-releases/2020/06/ acog-statement-on-covid-19-and-pregnancy

9. Ellington S, Strid P, Tong VT, et al. Characteristics of women of reproductive age with laboratory-confirmed SARS-CoV-2 infection by pregnancy status - United States, January 22-June 7, 2020. MMWR Morb Mortal Wkly Rep 2020; 69(25):769-775. doi:10.15585/ mmwr.mm6925a1

10. Calabrese C. COVID-19 and your rheumatology patients. Cleve Clin J Med 2020 May 14. doi:10.3949/ccjm.87a.ccc027

11. Centers for Disease Control and Prevention. Interim guidance for rapid antigen testing for SARS-CoV-2. Updated September 4, 2020. Accessed October 7, 2020. https://www.cdc.gov/coronavirus/2019ncov/lab/resources/antigen-tests-guidelines.html

12. Kucirka, L, et al. Variation in False-Negative Rate of Reverse Transcriptase Polymerase Chain Reaction-Based SARS-CoV-2 Tests by Time Since Exposure. Ann. Intern. Med. 2020; 173(4):262-267. doi. org/10.7326/M20-1495

13. Marty, F, et al. How to Obtain a Nasopharyngeal Swab Specimen. N Engl J Med 2020; 382:e76. DOI: 10.1056/NEJMvcm2010260

14. Simpson S, Kay FU, Abbara S, et al. Radiological Society of North America expert consensus statement on reporting chest CT findings related to COVID-19. Endorsed by the Society of Thoracic Radiology, the American College of Radiology, and RSNA - secondary publication. J Thorac Imaging 2020; 35(4):219-227. doi:10.1097/ RTI.0000000000000524

15. Wang X, Ferro EG, Zhou G, Hashimoto D, Bhatt DL. Association between universal masking in a health care system and SARS-CoV-2 positivity among health care workers. JAMA 2020; 324(7):703-704. doi:10.1001/jama.2020.12897

16. van Doremalen N, Bushmaker T, Morris DH, et al. Aerosol and surface stability of SARS-CoV-2 as compared with SARS-CoV-1. N Engl J Med 2020; 382(16):1564-1567. doi:10.1056/NEJMc2004973
17. Kampf G, Todt D, Pfaender S, Steinmann E. Persistence of coronaviruses on inanimate surfaces and their inactivation with biocidal agents. J Hosp Infect 2020; 104(3):246-251. doi:10.1016/j. jhin.2020.01.022

18. Laktineh A, Daoud A, Dhar S, Engles L, Soubani AO. What mask should I wear to protect against transmissible acute respiratory infections? Cleve Clin J Med 2020; 87(5):262-265. doi:10.3949/ ccjm.87a.19057

19. Centers for Disease Control and Prevention. Using PPE. Updated August 19, 2020. Accessed October 7, 2020. https://www.cdc.gov/ coronavirus/2019-ncov/hcp/using-ppe.html

20. New extended-use and re-use PPE guidelines; Cleveland Clinic Caregiver Communications, April 6, 2020

21. Lippi G, Plebani M. Laboratory abnormalities in patients with COVID-2019 infection. Clin Chem Lab Med 2020; 58(7):1131-1143. doi:10.1515/cclm-2020-0198

22. Day M. Covid-19: ibuprofen should not be used for managing symptoms, say doctors and scientists. BMJ 2020; 368:m1086

23. World Health Organization. The use of non-steroidal antiinflammatory drugs (NSAIDs) in patients with COVID-19. April 19, 2020. Accessed October 8, 2020. hacttp:WHO/2019-nCoV/Sci_Brief/ NSAIDs/2020.1

24. Bozkurt B, Kovacs R, Harrington B. HFSA/ACC/AHA statement addresses concerns re: using RAAS antagonists in COVID-19. ACC News Story March 17, 2020. Accessed October 8, 2020. https://bit. ly/2UiMyt6

25. Kow CS, Hassan SS. Meta-analysis of effectiveness of statins in patients with severe COVID-19. Am J Cardiol 2020 Aug 12;S00029149(20)30823-7. doi:10.1016/j.amjcard.2020.08.004

26. Zhu L, She Z-G, Cheng X, et al. Association of blood glucose control and outcomes in patients with COVID-19 and pre-existing type 2 diabetes. Cell Metab 2020; 31:1068-1077.e3. doi:10.1016/j. cmet.2020.04.021

27. Singh AK, Singh $\mathbf{R}$. Is metformin ahead in the race as a repurposed host-directed therapy for patients with diabetes and COVID19? Diabetes Res Clin Pract 2020 Jul; 165:108268. doi:10.1016/j. diabres.2020.108268108268, JULY 01, 2020

28. Zhou K, Al-Jaghbeer M, Lansang MC. Hyperglycemia management in hospitalized patients with COVID-19. Cleve Clin J Med $2020 \mathrm{Jul}$ 17. doi:10.3949/ccjm.87a.ccc012

29. Prasad M, Visrodia K. Should I prone non-ventilated awake patients? Cleve Clin J Med June 2020 Jun 30. doi:10.3949/ccjm.87a. ccc050

30. Thompson AE, Ranard BL, Wei $\mathbf{Y}$, Jelic S. Prone positioning in awake, nonintubated patients with COVID-19 hypoxemic respiratory failure. JAMA Intern Med 2020; e203030. doi:10.1001/ jamainternmed.2020.3030

31. Llitjos JF, Leclerc M, Chochois $\mathbf{C}$, et al. High incidence of venous thromboembolic events in anticoagulated severe COVID-19 patients. J Thromb Haemost 2020; 18(7):1743-1746. doi:10.1111/ jth.14869

32. Lodigiani C, lapichino G, Carenzo L, et al. Venous and arterial thromboembolic complications in COVID-19 patients admitted to an academic hospital in Milan, Italy. Thromb Res 2020 Jul; 191:9-14. doi:10.1016/j.thromres.2020.04.024

33. Artifoni M, Danic G, Gautier G et al. Systematic assessment of venous thromboembolism in COVID-19 patients receiving thromboprophylaxis: incidence and role of D-dimer as predictive factors. J. Thromb Thrombolysis 2020; 50(1):211-216. doi:10.1007/s11239-020-02146-z

34. Nadkarni GN, Lala A, Bagiella E, et al. Aniticoagulation, mortality, bleeding and pathology among patients hospitalized with COVID-19: a single health system study. J Am Coll Cardiol 2020 Aug 24;S0735-1097(20)36408-1. doi:10.1016/j.jacc.2020.08.041

35. Spyropoulos AC, Lipardi C, Xu J, et al. Modified IMPROVE VTE risk score and elevated D-dimer identify a high venous thromboembolism risk in acutely ill medical population for extended thromboprophylaxis. TH Open 2020; 4(1):e59-e65. doi:10.1055/s-0040-1705137

36. Mucha SR, Dugar S, McCrae K, Joseph D, Bartholomew J, Sacha GL, Militello M. Coagulopathy in COVID-19: manifestations and 
management. Cleve Clin J Med 2020; 87(8):461-468. doi:10.3949/ ccjm.87a.ccc024

37. Moores LK, Tritschler T, Brosnahan S, et al. Prevention, diagnosis and treatment of VTE in patients with coronavirus disease 2019: Chest guideline and expert panel report. Chest 2020; 158(3):11431163. doi:10.1016/j.chest.2020.05.559

38. RECOVERY Collaborative Group; Horby P, Lim WS, Emberson JR, et al. Dexamethasone in hospitalized patients with COVID-19 preliminary report. N Engl J Med 2020 Jul 17; NEJMoa2021436. doi:10.1056/NEJMoa2021436

39. WHO Rapid Evidence Appraisal for COVID-19 Therapies (REACT) Working Group; Sterne JAC, Murth S, Diaz J, et al. Association between administration of systemic corticosteroids and mortality among critically ill patients with COVID-19: a meta-analysis. JAMA 2020; 324(13):1-13. doi:10.1001/jama.2020.17023

40. Beigel JH, Tomashek KM, Dodd LE, et al. Remdesivir for the treatment of COVID-19 - preliminary report. N Engl J Med 2020 May 22;NEJMoa2007764. doi:10.1056/NEJMoa2007764

41. Spinner CD, Gottlieb RL, Criner GJ, et al. Effect of remdesivir vs standard care on clinical status at 11 days in patients with moderate COVID-19. JAMA 2020; 324(11):1048-1057. doi:10.1001/ jama.2020.16349

42. Food and Drug Administration. FDA news release. COVID-19 update: FDA broadens emergency use authorization for Veklury (remdesivir) to include all hospitalized patients for treatment of COVID-19. August 28, 2020. Accessed October 8, 2020. https:// www.fda.gov/news-events/press-announcements/covid-19-updatefda-broadens-emergency-use-authorization-veklury-remdesivirinclude-all-hospitalized

43. Food and Drug Administration. FDA news release. FDA approves first treatment for COVID-19. October 22, 2020. Accessed October 23, 2020. https://www.fda.gov/news-events/press-announcements/ fda-approves-first-treatment-covid-19

44. Shen C, Wang Z, Zhao F, et al. Treatment of 5 critically ill patients with COVID-19 with convalescent plasma. JAMA 2020; 323(16):1582-1589. doi:10.1001/jama.2020.478

45. Duan K, Liu B, Li C, et al. The feasibility of convalescent plasma therapy in severe COVID-19 patients: a pilot study. Medrxiv 2020 March 16. doi:10.1101/2020.03.16.20036145

46. Li L, Zhang W, Hu Y, et al. Effect of convalescent plasma therapy on time to clinical improvement in patients with severe and lifethreatening COVID-19: a randomized clinical trial. JAMA 2020; 324(5):460-470. doi:10.1001/jama.2020.1004

47. Joyner MJ, Senefeld JW, Klassen SA, et al. Effect of convalescent plasma on mortality among hospitalized patients with COVID-19: initial three-month experience. medRxiv 2020; 2020.08.12.20169359. doi:10.1101/2020.08.12.20169359

48. U.S. National Library of Medicine. COVID-19 studies from the World Health Organization database. Accessed October 8, 2020. https:// clinicaltrials.gov/ct2/who_table

49. Medina M, Babiuch C, Card M, et al. Home monitoring for COVID19 Cleve Clin J Med 2020 Jun 11. doi:10.3949/ccjm.87a.ccc028

50. Centers for Disease Control and Prevention. Ending home isolation. Interim guidance. Updated July 20, 2020. Accessed October 8, 2020. https://www.cdc.gov/coronavirus/2019-ncov/hcp/dispositionin-home-patients.html

51. Bajaj NS, Vaduganathan M, Qamar A, et al. Extended prophylaxis for venous thromboembolism after hospitalization for medical illness: a trial sequential and cumulative meta-analysis. PLoS Med 2019; 16(4):e1002797. doi:10.1371/journal.pmed.1002797

52. Spyropoulos AC, Lipardi C, Xu J, et al. Modified IMPROVE VTE risk score and elevated D-dimer identify a high venous thromboembolism risk in acutely ill medical population for extended thromboprophylaxis. TH Open 2020; 4(1):e59-e65. doi:10.1055/s-0040-1705137

53. Bhimraj A, Morgan RL, Shumaker AH, et al. Infectious Diseases Society of America guidelines on the treatment and management of patients with COVID-19. Updated September 25, 2020. Accessed October 8, 2020. https://www.idsociety.org/practice-guideline/ covid-19-guideline-treatment-and-management/
54. National Institutes of Health. COVID-19 treatment guidelines. What's new in the guidelines. Updated September 1, 2020. Accessed October 8, 2020. https://www.covid19treatmentguidelines.nih.gov/ whats-new/

55. Centers for Disease Control and Prevention. Hospitalization surveillance network COVID-NET. Updated August 28, 2020. Accessed October 8, 2020. https://www.cdc.gov/coronavirus/2019-ncov/coviddata/covid-net/purpose-methods.html

Correspondence: Maria Miklowski, MD, Department of Hospital Medicine, Cleveland Clinic, M2 Annex, 9500 Euclid Avenue, Cleveland, OH 44195; miklowm@ccf.org 